\title{
Crystal structure of $\mathrm{C}$-terminal desundecapeptide nitrite reductase from Achromobacter cycloclastes
}

\author{
Hai-Tao Li ${ }^{\text {a }}$, Tschining Chang ${ }^{\mathrm{b}}$, Wen-Chang Chang ${ }^{\mathrm{b}, \mathrm{c}}$, Chung-Jung Chen ${ }^{\mathrm{d}}$, \\ Ming-Yih Liu ${ }^{\text {d,* }}$, Lu-Lu Gui ${ }^{\text {a }}$, Ji-Ping Zhang ${ }^{\text {a }}$, Xiao-Min An ${ }^{\text {a }}$, Wen-Rui Chang ${ }^{\text {a,* }}$ \\ a National Laboratory of Biomacromolecules, Institute of Biophysics, Chinese Academy of Sciences, 15th Datun Road, Chaoyang District, \\ Beijing 100101, China \\ ${ }^{\mathrm{b}}$ Institute of Biological Chemistry, Academia Sinica, Taipei 11529, Taiwan \\ ${ }^{\mathrm{c}}$ Institute of Biochemical Sciences, National Taiwan University, Taipei 10629, Taiwan \\ ${ }^{\mathrm{d}}$ Life Science Group, Research Division, National Synchrotron Radiation Research Center, Hsinchu 30076, Taiwan
}

Received 27 September 2005

Available online 15 November 2005

\begin{abstract}
Monoclinic crystal structure of C-terminal desundecapeptide nitrite reductase (NiRc-11) from Achromobacter cycloclastes was determined at $2.6 \AA$. NiRc-11 exists as a loose trimer in the crystal. Deletion of 11 residues eliminates all intersubunit hydrogen bonds mediated by the C-terminal tail. The rigid irregular coil 105-112, which constitutes part of the sidewall of the active site pocket, undergoes conformational changes and becomes highly flexible in NiRc-11. Correspondingly, the linker segments between the two copper sites 95-100 and 135-136 are partly relaxed in conformation, which leads to disrupted active site microenvironments responsible for the activity loss and spectral change of NiRc-11. Comparison with the native structure revealed a bulky residue Met331 fastened by hydrogen bonding, which may play a direct role in keeping the right copper site geometry by protruding its side chain against the irregular coil 105-112. Sequence alignment showed that the bulky residue is conserved at position 331, indicating an equal importance of C-terminal segment in other copper-containing nitrite reductases.
\end{abstract}

(C) 2005 Elsevier Inc. All rights reserved.

Keywords: Crystal structure; Nitrite reductase; C-terminal truncation; Copper coordination; Trimer formation

Copper-containing nitrite reductase (CuNIR) is an important metalloprotein involved in the denitrification pathway. It catalyzes the reduction of nitrite to gaseous nitric oxide, leading to the direct loss of fixed nitrogen from the terrestrial environment. So far more than $15 \mathrm{CuNiRs}$ have been well characterized from a variety of organisms including bacteria, actinomycete, archeabacteria, and even fungi. The structure-function relationships of these proteins have been extensively explored with a combination of some physicochemical methods and protein engineering techniques $[1,2]$.

\footnotetext{
${ }^{*}$ Corresponding authors. Fax: +88635783813 (M.-Y. Liu); +86 10 64889867 (W.-R. Chang).

E-mail addresses: mingliu@nsrrc.org.tw (M.-Y. Liu), wrchang@ sun5.ibp.ac.cn (W.-R. Chang).
}

AcNiR isolated from Achromobacter cycloclastes is the first $\mathrm{CuNiR}$ whose crystal structure has been determined $[3,4]$. Thereafter, crystal structures of CuNiRs from Alcaligenes faecalis [5], Alcaligenes xylosoxidans [6,7], Neisseria gonorrhoear [8], and Rhodobacter sphaeroides [9] were also reported. It is shown that $\mathrm{CuNiR}$ is organized as a homotrimer with each monomer comprised of two cupredoxin domains. A total of six copper atoms are found in the trimer and can be further classified into two types. The type 1 copper $(\mathrm{T} 1 \mathrm{Cu})$ is buried in domain I of each monomer, being ligated by residues His95, Cys136, His145, and Met150 (AcNiR numbering); it servers as the chromophoric center and the site for outer electron transfer in CuNiR. The type 2 copper $(\mathrm{T} 2 \mathrm{Cu})$ is located at the intermonomer cleft and is the active center for nitrite reduction. It is coordinated by one exogenous solvent ligand and three 
histidine residues: His100, His135, and His306, in which His 306 comes from domain II of an adjacent monomer. The two copper sites are approximately $12.5 \AA$ apart and are close related through linker segments His95-His 100 and His135-Cys136, which provides a well-engineered configuration for high efficient electron transfer and nitrite reduction $[2,6,10]$. Two water-bridged residues near the $\mathrm{T} 2 \mathrm{Cu}$ site, Asp98 and His 255 , are catalytically critical residues. Mutational and structural studies showed that they are involved in substrate anchoring, electron transfer as well as nitrite reduction [11-13].

The trimeric state of $\mathrm{CuNiR}$ has been established by $\mathrm{X}$-ray crystallography, sedimentation equilibrium measurement [3], and solution X-ray scattering techniques [14]. The AcNiR trimer is very stable and shows resistance to SDS at room temperature even when the SDS concentration is as high as $4 \%(\mathrm{w} / \mathrm{v})$ [15]. A similar property was also observed in the $\mathrm{CuNiR}$ from A. faecalis [16]. Crystal structure studies reveal an unusual but conserved elongated C-terminal tail in AcNiRs. It extends from one monomer to another, forming $\beta$-sheet hydrogen bonds between monomers, and was considered a key factor for maintaining the AcNiR trimer [3]. We have generated a series of C-terminally deleted AcNiR mutants to explore the role of $\mathrm{C}$-terminal tail in AcNiR. It was shown that the C-terminal tail is essential for maintaining not only the quaternary structure but also the enzyme activity of AcNiR [15]. Desundecapeptide nitrite reductase (NiRc-11) is the AcNiR analogue with 11 residues (330-340) deleted from the C-terminus. Compared to the despentapeptide AcNiR (NiRc-5), which remains as a rigid trimer and retains $72 \%$ enzyme activity, NiRc-11 undergoes drastic changes in its biochemical property as it exhibits distorted optical spectrum and loses all its enzyme activity as well as its resistance to SDS. We have reported the crystal structure study of NiRc-5, which revealed a perturbed $\mathrm{T} 2 \mathrm{Cu}$ site microenvironment after the pentapeptide deletion, although no significant structural changes occur [17]. To further elucidate the function of the $\mathrm{C}$-terminal tail in $\mathrm{CuNiR}$, we determined the monoclinic crystal structure of NiRc-11 at $2.6 \AA$.

\section{Materials and methods}

Data collection and processing. Mutagenesis, purification, and crystallization of NiRc-11 have been described previously [15,18]. Using the monoclinic crystal growing at $\mathrm{pH} 5.4$, a new data set was collected at $100 \mathrm{~K}$ on the Taiwan contract beamline BL12B2 at SPring8, Japan, with an Q-4R CCD detector (ADSC). A total of $360 \times 1^{\circ}$ frames were collected and the final data set was processed to $2.6 \AA$ using program $H K L 2000$ [19]. The crystal belongs to the $P 2_{1}$ space group with cell parameters: $a=85.8 \AA, b=111.1 \AA, c=122.9 \AA$, and $\beta=101.7^{\circ}$. The overall $R$ merge, completeness, and signal-to-noise ratio of the new data set between resolution 20 and $2.6 \AA$ are $13.4 \%, 99.5 \%$, and 14.8 , respectively (Table 1).

Structure determination and refinement. Initial phasing of NiRc-11 monoclinic crystal was achieved by the molecular replacement method using the program Amore [20]. Native AcNiR trimer (PDBid: 1NIA) was used as the searching model after deleting its $\mathrm{C}$-terminal 11 residues and all solvent and copper atoms. This yielded two distinct solutions corresponding to two trimers in one asymmetrical unit of the crystal as

Table 1

Data collection and structure refinement statistics of NiRc-11 structure

Data collection

Space group

Unit cell dimension

Resolution range $(\AA)$ (last shell)

No. of observations

Unique reflections

$R_{\text {merge }}(\%)$ (last shell)

$I / \sigma I$ (last shell)

Completeness (\%) (last shell)

$P 2_{1}$

$a=85.8 \AA, b=111.1 \AA, c=122.9 \AA$,
$\beta=101.7^{\circ}$

$20-2.6(2.69-2.60)$

496,045

69,377

$13.4(65.6)$

$14.8(3.4)$

$99.5(98.1)$

Structure refinement

Resolution $(\AA)$

$R$-work/ $R$-free (\%)

r.m.s.d. bonds $(\AA) /$ angles $\left({ }^{\circ}\right)$

Number of residues/waters

Average $B$-factors

Main chain $\left(\AA^{2}\right)$

Side chain $\left(\AA^{2}\right)$

Water $\left(\AA^{2}\right)$

Ramachandran plot

Most favored region

Additional allowed region

Generous allowed region
$20-2.6$
$18.2 / 21.7$
$0.006 / 1.56$
$1904 / 376$
31.4
32.7
27.7
$87.6 \%$
$12.3 \%$
$0.1 \%$

supposed by the cell content analysis. The following structure refinement was carried out using program CNS [21] with the maximum likelihood targets. About $4 \%$ of the total reflections were picked out for cross-validation using program DATAMAN based on a "thin-shell" method [22]. During the refinement, no constraints were applied to the coordination geometry of copper atoms; strict and restrained non-crystallographic symmetry (NCS) were applied in earlier steps of the refinement and were released at later stages. All model buildings and adjustments were performed manually using the O program [23] on an SGI workstation. Twelve copper atoms were added based on the strong difference map. Six chloride ions instead of waters were modeled as the T2Cu solvent ligands, because clear positive difference density inevitably existed when water was modeled even if the $B$-factor was refined to as low as $1.0 \AA^{2}$. After several cycles of refinement and model building, the model was finally refined to $R$-factor 0.182 and $R$-free 0.217 . The refinement statistics of NiRc-11 structure is summarized in Table 1. Coordinate files and structure factors of NiRc-11 have been deposited into Protein Data Bank with entry code 2AVF.

Copper content measurement. The copper content of NiRc-11 was measured to verify the occupancy of $\mathrm{T} 2 \mathrm{Cu}$ in crystal. The copper concentration was determined with the plasma emission spectrophotometer. The protein concentration was determined using the Lowry method [24].

\section{Results and discussions}

\section{Overall structure}

The crystal structure of NiRc-11 was solved at $2.6 \AA$. Despite the loss of SDS resistance and enzyme activity, NiRc-11 still exists as a trimer in the crystal. There are two trimers in the final refined model. Among the six polypeptide chains, residue A12-325, B11-326, C10-325, D5-324, E10-324, and F5-327 from a full length of 329 residues are modeled according to the electron density map. Similar to a native enzyme, NiRc-11 trimer comprised of three monomers arranged head to tail around a threefold axis (Fig. 1A). The deletion of 11 residues did 

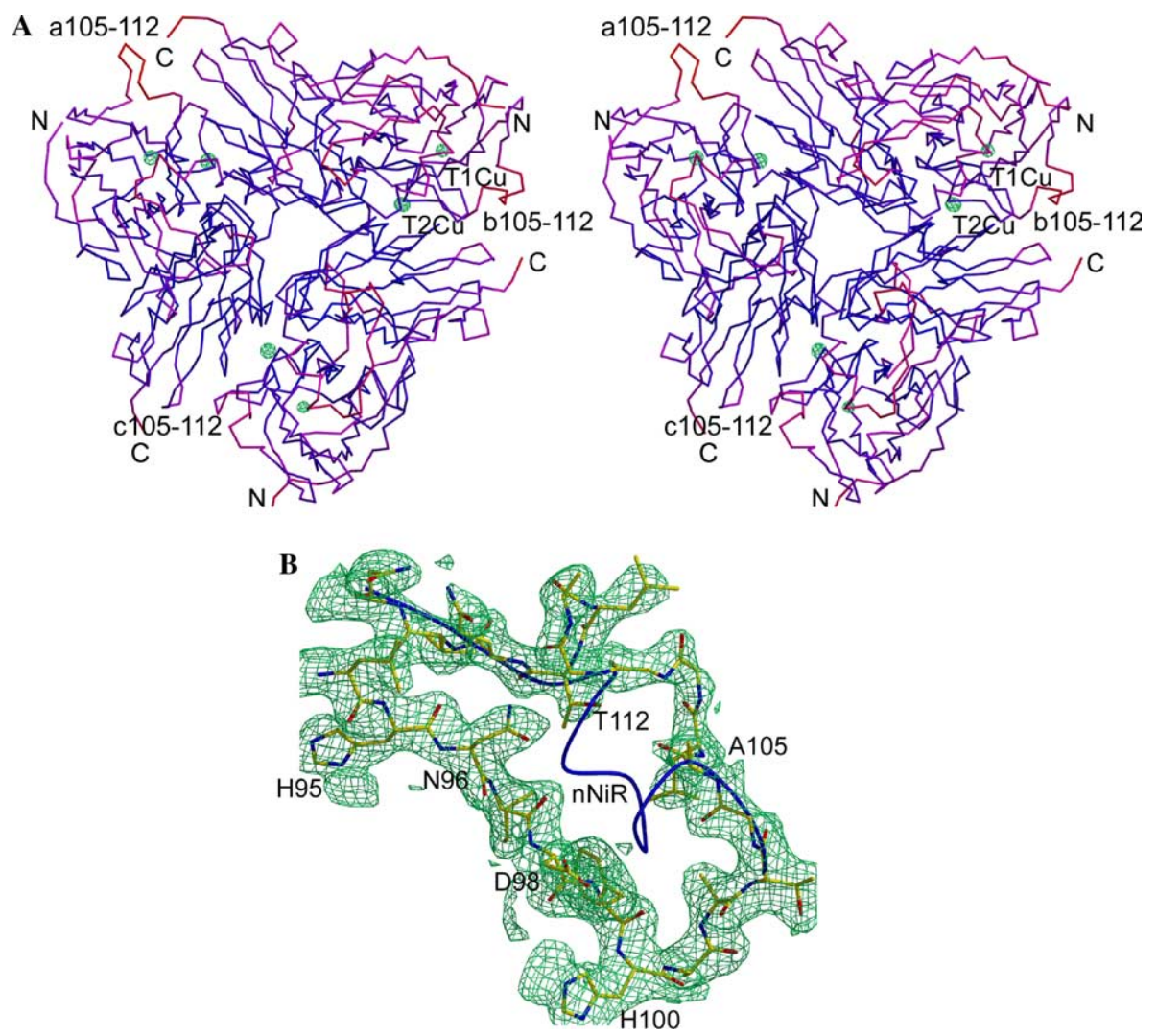

Fig. 1. (A) $B$-factor represented C $\alpha$ trace stereoview of NiRc-11 trimer. The trimer is colored by ramping from blue $\left(B \leqslant 15 \AA^{2}\right)$ to red $\left(B \geqslant 70 \AA^{2}\right)$ based on the $B$ value of each residue; the copper atoms are shown in their fo-fc electron density (at $15 \sigma$ ) calculated against the final model after deleting all copper atoms. (B) Conformational change of irregular coil b105-112 in NiRc-11. The blue coil represents the conformation of b105-112 in native AcNiR (nNiR). The 2 fo-fc omit map is contoured at $0.8 \sigma$ and residues b105-112 is omitted in map calculation. Figures were produced with Molscript [27] and Raster3D [28]. (For interpretation of the references to color in this figure legend, the reader is referred to the web version of this paper.)

not affect the core structure of AcNiR; all $\beta$-strands and $\alpha$ helixes that are necessary to form the two cupredoxin $\beta$ barrel domains are well retained in NiRc-11. The C $\alpha$-based superimposition of NiRc-11 trimer with native enzyme (PDBid: 2NRD) gives an RMSD around $0.44 \AA^{2}$. Major conformational changes in NiRc-11 occur at the C-terminal tail, an irregular coil 105-112, and the two copper site linker segments; other intrinsic flexible regions such as the N-terminal tail, the "tower loop" and the "domain linker" loop at the protein surface also show certain differences, but do not change their overall conformations.

There are altogether 12 copper atoms in the two NiRc11 trimers. Six chloride ions, which may be introduced during protein purification, take the place of water and become the solvent ligand to $\mathrm{T} 2 \mathrm{Cu}$. The $\mathrm{T} 2 \mathrm{Cu}$ at the interface of two subunits has been prone to be lost during enzyme preparation or other drastic conditions. It was reported that the specific activity of $\mathrm{CuNiR}$ is directly proportional to the occupancy of $\mathrm{T} 2 \mathrm{Cu}$ [25]. However, despite the activity loss, all copper atoms in NiRc-11 are well retained as revealed by their strong electron densities. In addition, as in the NiRc- 5 structure, the $\mathrm{T} 2 \mathrm{Cu}$ in NiRc11 shows better electron densities than the $\mathrm{T} 1 \mathrm{Cu}$ that is buried in each monomer (Fig. 1A). The refined $B$-factors of the $\mathrm{T} 2 \mathrm{Cu}$ give an average value of $\sim 19.9 \AA^{2}$, which is considerably lower than a value of $\sim 29.6 \AA^{2}$ at the $\mathrm{T} 1 \mathrm{Cu}$ sites. These results strongly suggest a full occupancy of the $\mathrm{T} 2 \mathrm{Cu}$ in NiRc-11. Copper content measurement was performed to verify this result, and yielded a molecular ratio of $\sim 6.0$ between copper atoms and one NiRc-11 trimer. This confirms the crystallographic result of the copper occupancy, and demonstrates that the activity loss of NiRc-11 is not caused by the loss of the T2Cu. Meanwhile, the possibility of metal ion substitution at the $\mathrm{T} 2 \mathrm{Cu}$ site, such as by zinc, is also excluded by the copper content measurement.

\section{C-terminal tail}

As shown in Fig. 1A, the C-terminal tail of NiRc-11 becomes highly flexible. Some residues at the end cannot be modeled due to their poor electron densities. Whereas, in the native structure, the C-terminal segment represents a well-ordered region as it mediates extensive interactions between subunits via an extended $\beta$-strand motif. In NiRc-11, residue deletion eliminates the whole $\beta$-strand and causes a loss of all intersubunit hydrogen bonds mediated by the C-terminal tail. Without the contribution by the C-terminal segment, NiRc-11 is no more a tightly associated trimer, which is further proved by the high $B$-factor 
distribution at the subunit interface. Biochemical analysis showed that NiRc-11 loses its resistance to the denaturing reagent SDS, whereas native AcNiR and NiRc-5 do not. In the case of $\mathrm{NiRc}-5$, the extended $\beta$-sheet interaction between monomers is still retained, although nearly half the hydrogen bonds are lost [17]. On basis of these results, it can be inferred that the extended $\beta$-sheet intersubunit interactions play a key role in ensuring the rigidity of the AcNiR trimer, though it may not be essential for the trimer formation.

\section{Irregular coil 105-112}

The conformational change of the irregular coil 105-112 is an unexpected feature in the NiRc-11 structure. In the native enzyme and NiRc-5, the irregular coil is a rigid structural element; it is kept in crouched conformation, leans forward against the linker segment 95-100, and constitutes part of the sidewall of the active site pocket around the T2Cu site. As illustrated in Fig. 1B, the irregular coil in NiRc-11 stretches out from its original position and takes on a more relaxed conformation. It is also shown in Fig. 1A that the irregular coil is no longer well ordered; instead, it becomes one of the most flexible regions in NiRc-11. The irregular coils in different subunit of the two NiRc-11 trimers have different conformations except for the high $B$-factor distribution. In chain $\mathrm{C}$, the irregular coil is very similar to that of the native protein. While in other chains, the irregular coils adopt much different conformations, among which the irregular coil of chain A represents the most stretched (Fig. 1A). Differences in the conformation of the irregular coils are related to the different crystal packing environments. For example, the irregular coil of chain $\mathrm{C}$ is close to the $\mathrm{N}$-terminal segment of chain $\mathrm{A}$ in one symmetry-related trimer; this proximity may impose additional constraints on the irregular coil and keep it in the crouched state. The relaxation of the irregular coil 105-112 is a characteristic of NiRc-11. It can be inferred from the solved crystal structures that the irregular coil 105-112 is constrained in AcNiR and NiRc5. Deletion of the eleven residues in NiRc-11 not only abolishes the intersubunit interaction mediated by the Cterminal tail but also releases the constraints imposed on the irregular coil. Since the deletion of five residues in NiRc-5 did not cause the relaxation of the irregular coil, the structural element responsible for the constraining can be further mapped to peptide segment $330-335$ in the C-terminal tail of AcNiR.

\section{Copper site linker segments}

In $\mathrm{CuNiR}$, the $\mathrm{T} 1 \mathrm{Cu}$ and the $\mathrm{T} 2 \mathrm{Cu}$ are close linked by two bridging segments His95-His100 and His135-Cys136, which provide half the copper ligating residues and a catalytic residue Asp98 [2]. Fig. 2 shows the superimposed copper sites between native AcNiR and NiRc-11. It is clear that the two linker segments undergo considerable changes
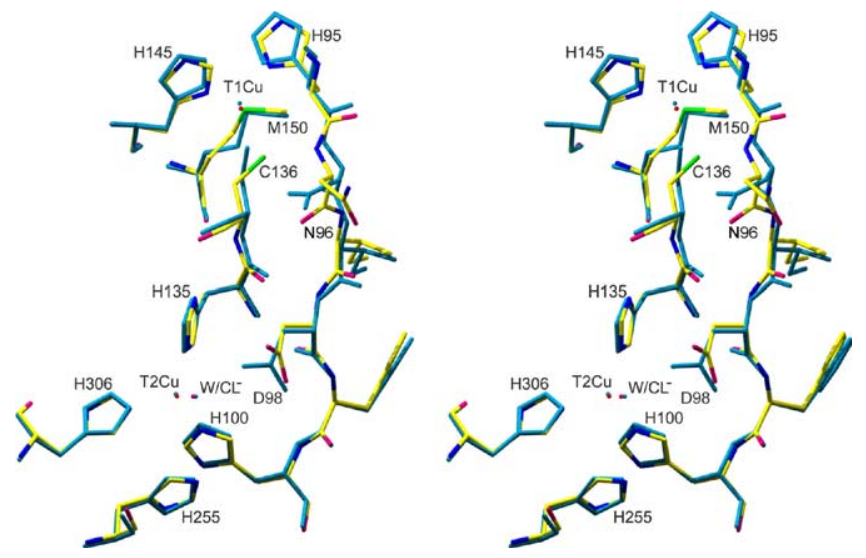

Fig. 2. Superimposed copper sites between NiRc-11 and native enzyme. Coordinates are taken from chain B of NiRc-11 (mixed color) and PDB entry 2NRD of AcNiR (cyan). Superimposition is performed using Spdbviewer [29] based on the residues shown. (For interpretation of the references to color in this figure legend, the reader is referred to the web version of this paper.)

and take on partly relaxed conformations. The most remarkable conformational change is the flipping of Asn96. As shown in Fig. 2, the main chain carbonyl group of Asn96 in NiRc-11 rotates outwards by $\sim 80^{\circ}$; the main chain $\mathrm{C} \alpha$ atom shifts upwards by $\sim 1.1 \AA$; and the side chain adopts a new rotamer conformation occupying the space left by the relaxation of the irregular coil. Accordingly, the T1Cu ligating residue His95 displays an overall shift so as to fit the flipping of Asn96. Meanwhile, the catalytic residue Asp98 also shifts slightly towards Asn96, followed by a noticeable side chain rotation, which results in a departure of the proton attracting oxygen atom from the chloride ion ligand of the $\mathrm{T} 2 \mathrm{Cu}$. Likewise, residue Cys136 in the second segment shows a side chain rotation towards Asn96; its torsion angle $\chi 1$ changes from $+178^{\circ}$ in the native enzyme to $-133^{\circ}$ in NiRc-11 (chain B). The conformational change of Asn96 is closely related to the relaxation of the irregular coil 105-112. In chain C of NiRc-11, the irregular coil $105-112$ is in the same crouched state as that of the native enzyme; as a result, no flipping of Asn96 is observed in chain C. On the other hand, the rotation of the Cys136 side chain is most likely a consequence of the flipping of Asn96, as the flipping of Asn96 could set aside additional space for the rotation of the Cys136 side chain. Correspondingly, residue Cys136 in chain $\mathrm{C}$ shows a $\chi 1$ value of $-159^{\circ}$, which is the least altered conformation of the Cys136 side chain among the six monomers in one asymmetric unit.

\section{Copper sites geometries and activity loss}

As revealed in Fig. 2 and Table 2, the copper site geometries are distorted following the conformational changes of the two linker segments. Interestingly, the distortion mainly happens to the $\mathrm{T} 1 \mathrm{Cu}$ center of NiRc-11, despite that the $\mathrm{T} 2 \mathrm{Cu}$ center is formed at the monomer-monomer interface of a loose trimer. Following the conformational 
Table 2

Copper sites geometries

\begin{tabular}{|c|c|c|}
\hline & NiRc-11 & $\mathrm{AcNiR}^{\mathrm{a}}$ \\
\hline $\begin{array}{l}\text { T1Cu bond length }(\AA) \\
\mathrm{H}^{2} \mathrm{~N}^{\delta 1} \\
\mathrm{C} 136 \mathrm{~S}^{\gamma} \\
\mathrm{H} 145 \mathrm{~N}^{\delta 1} \\
\mathrm{M}^{\delta} 50 \mathrm{~S}^{\delta}\end{array}$ & $\begin{array}{l}2.21(0.04)^{\mathrm{b}} \\
2.37(0.08) \\
2.08(0.16) \\
2.32(0.06)\end{array}$ & $\begin{array}{l}2.02 \\
2.16 \\
2.02 \\
2.59\end{array}$ \\
\hline $\begin{array}{l}\text { T1Cu bond angles }\left(^{\circ}\right) \\
\mathrm{H}^{\circ} 5 \mathrm{~N}^{\delta 1}-\mathrm{Cu}-\mathrm{C} 136 \mathrm{~S}^{\gamma} \\
\mathrm{H} 95 \mathrm{~N}^{\delta 1}-\mathrm{Cu}-\mathrm{H} 145 \mathrm{~N}^{\delta 1} \\
\mathrm{H} 95 \mathrm{~N}^{\delta 1}-\mathrm{Cu}-\mathrm{M} 150 \mathrm{~S}^{\delta} \\
\mathrm{C} 136 \mathrm{~S}^{\gamma}-\mathrm{Cu}-\mathrm{H} 145 \mathrm{~N}^{\delta 1} \\
\mathrm{C}^{\gamma} 36 \mathrm{~S}^{\gamma}-\mathrm{Cu}-\mathrm{M} 150 \mathrm{~S}^{\delta} \\
\mathrm{H} 145 \mathrm{~N}^{\delta 1}-\mathrm{Cu}-\mathrm{M} 150 \mathrm{~S}^{\delta}\end{array}$ & $\begin{array}{l}115(4.9) \\
101(3.3) \\
89(2.1) \\
118(6.8) \\
103(6.3) \\
127(7.4)\end{array}$ & $\begin{array}{r}134 \\
99 \\
88 \\
103 \\
108 \\
129\end{array}$ \\
\hline $\begin{array}{l}\text { T2Cu bond length }(\AA) \\
\mathrm{H} 100 \mathrm{~N}^{\varepsilon 2} \\
\mathrm{H} 135 \mathrm{~N}^{\varepsilon 2} \\
\mathrm{H} 306 \mathrm{~N}^{\varepsilon 2} \\
\mathrm{Cl}^{-} / \mathrm{H}_{2} \mathrm{O}\end{array}$ & $\begin{array}{l}2.05(0.12) \\
2.09(0.09) \\
2.09(0.09) \\
2.13(0.06)\end{array}$ & $\begin{array}{l}2.00 \\
2.04 \\
2.19 \\
1.90\end{array}$ \\
\hline $\begin{array}{l}\text { T2 } \mathrm{Cu} \text { bond angles }\left({ }^{\circ}\right) \\
\mathrm{H} 100 \mathrm{~N}^{\varepsilon 2}-\mathrm{Cu}-\mathrm{H} 135 \mathrm{~N}^{\varepsilon 2} \\
\mathrm{H} 100 \mathrm{~N}^{\varepsilon 2}-\mathrm{Cu}-\mathrm{H} 306 \mathrm{~N}^{\varepsilon 2} \\
\mathrm{H} 135 \mathrm{~N}^{\varepsilon 2}-\mathrm{Cu}-\mathrm{H} 306 \mathrm{~N}^{\varepsilon 2} \\
\mathrm{Cl}^{-} / \mathrm{H}_{2} \mathrm{O}-\mathrm{Cu}-\mathrm{H} 100 \mathrm{~N}^{\varepsilon 2} \\
\mathrm{Cl}^{-} / \mathrm{H}_{2} \mathrm{O}-\mathrm{Cu}-\mathrm{H} 135 \mathrm{~N}^{\varepsilon 2} \\
\mathrm{Cl}^{-} / \mathrm{H}_{2} \mathrm{O}-\mathrm{Cu}-\mathrm{H} 306 \mathrm{~N}^{\varepsilon 2}\end{array}$ & $\begin{array}{l}109(4.6) \\
106(5.1) \\
110(2.9) \\
106(3.2) \\
113(5.7) \\
112(4.4)\end{array}$ & $\begin{array}{l}104 \\
108 \\
111 \\
101 \\
110 \\
121\end{array}$ \\
\hline $\begin{array}{l}\text { Other bond length }(\AA) \\
\text { D } 98 \mathrm{O}^{\delta 1}-\mathrm{Cl}^{-} / \mathrm{H}_{2} \mathrm{O} \\
\mathrm{H} 255 \mathrm{~N}^{\varepsilon 2}-\mathrm{Cl}^{-} / \mathrm{H}_{2} \mathrm{O}\end{array}$ & $\begin{array}{l}3.61(0.37) \\
3.54(0.09)\end{array}$ & $\begin{array}{l}2.50 \\
3.50\end{array}$ \\
\hline
\end{tabular}

a 2.1 A native AcNiR structure at pH 5.4 with PDB entry code 2NRD.

b Average values over all chains in one asymmetric unit are listed; values in parentheses are the standard deviations.

changes of His 95 and Cys136 described above, Met150 and His145 also undergo adaptive conformational changes (Fig. 2). The maximum deviations between NiRc-11 and native $\mathrm{AcNiR}$ at the $\mathrm{T} 1 \mathrm{Cu}$ center are around $0.27 \AA$ in bond length and $19^{\circ}$ in bond angle (Table2). The most striking change to the $\mathrm{T} 1 \mathrm{Cu}$ site geometry is that the axial ligand Met150 - which is usually a weak ligand in native enzyme - exhibits an enhanced bonding to $\mathrm{T} 1 \mathrm{Cu}$ by a $\sim 0.27 \AA$ reduction in bond length. Meanwhile, the two strong ligand residues, Cys136 and His95, display weakened coordinations to $\mathrm{T} 1 \mathrm{Cu}$ by a $\sim 0.2 \AA$ elongation in bond length. Such changes indicate a profound rearrangement of the $\mathrm{T} 1 \mathrm{Cu}$ coordination sphere in NiRc-11, and in turn emphasize the importance of the C-terminal tail in maintaining the right copper site geometry. Since the $\mathrm{T} 1 \mathrm{Cu}$ site is the chromophoric center of $\mathrm{CuNiR}$, the distortion of the $\mathrm{T} 1 \mathrm{Cu}$ geometry is consistent with the distorted optical spectrum of NiRc-11. In contrast to the $\mathrm{T} 1 \mathrm{Cu}$ site, the geometry of the $\mathrm{T} 2 \mathrm{Cu}$ site is fairly well retained (Fig. 2). The maximum deviations in bond length and bond angle except for the solvent ligand are around $0.1 \AA$ and $5^{\circ}$, respectively (Table2). Although His 306 comes from the adjacent subunit, its coordination to $\mathrm{T} 2 \mathrm{Cu}$ is not broken by the looseness of the NiRc-11 trimer; on the contrary, its interaction with $\mathrm{T} 2 \mathrm{Cu}$ is even enhanced as reflected by the decreased bond length (Table 2). This feature, in combination with the low $B$-factor and the strong electron density at the $\mathrm{T} 2 \mathrm{Cu}$ site, indicates that the coordination to $\mathrm{T} 2 \mathrm{Cu}$ by the two subunits becomes a major factor for the maintenance of the NiRc-11 trimer during the absence of the C-terminal segment.

In $\mathrm{CuNiR}$, the two copper centers are harmoniously organized through the two linker segments to carry out the reduction of nitrite. It has been reported that nitrite reduction in $\mathrm{CuNiR}$ proceeds by an ordered mechanism in which electron transfer from the $\mathrm{T} 1 \mathrm{Cu}$ to the $\mathrm{T} 2 \mathrm{Cu}$ site occurs after the binding of nitrite to the oxidized $\mathrm{T} 2 \mathrm{Cu}$ ion [10]. In this process, the linker segments His135-Cys136 and His95-His100 are considered as the route for the internal electron transfer and the sequence motif for sensing the status of the $\mathrm{T} 2 \mathrm{Cu}$ site via the proton attracting group Asp98, respectively. The conformational relaxation of the two linker segments not only results in the distortion of the $\mathrm{T} 1 \mathrm{Cu}$ site geometry, but also causes largely impaired hydrogen bonding between the catalytic residue Asp98 and the $\mathrm{T} 2 \mathrm{Cu}$ solvent ligand. As shown in Table 2, the D980 ${ }^{\delta 1}-\mathrm{Cl}^{-}$distance in NiRc-11 becomes $\sim 3.61 \AA$ in average, which is in sharp contrast with a value of $\sim 2.5 \AA$ in the native enzyme where a proper hydrogen bond is formed. Therefore, although the $\mathrm{T} 2 \mathrm{Cu}$ site geometry and the catalytic residue His 255 conformation are not much affected (Fig. 2), the active site microenvironments are totally disrupted in NiRc-11. Such a disruption has most likely blocked the internal electron transfer and ruined the catalyzing ability of Asp98, thus leading to the complete activity loss of NiRc-11.

\section{Sequential steric constraining on active site geometry}

The superimposition of NiRc-11 with native enzyme reveals three layers of constraints from the $\mathrm{C}$-terminal tail to the copper center. As illustrated in Fig. 3, the first layer of constraint exists between the $\mathrm{C}$-terminal tail and the irregular coil 105-112, where the C-terminal segment confines the latter through side chain exclusion. Steric clashes exist between Met331 and Leu106, and between Lys336 and Leu111 when the irregular coil is relaxed. The distance between Met331 $\mathrm{S}^{\delta}$ and the relaxed Leu106 $\mathrm{C} \alpha$ atom is only $\sim 1.2 \AA$ (chain B). The second and the third layers of constraints are formed among the irregular coil and the two copper site linker segments. In the native AcNiR, these structural elements are stacked from the surface to the inner side of the protein. Two layers of constraints at the interfaces are revealed by the steric clash between the carbonyl group of Gly109 in the crouched irregular coil and the side chain of the relaxed Asn96, and by the close contact between the carbonyl group of the restrained Asn96 and the $\mathrm{S}^{\gamma}$ atom of the relaxed Cys136 (Fig. 3). Therefore, following the red-colored residues shown in Fig. 3, a sterically constraining chain is formed and functions to maintain the optimal active site geometry. In NiRc-11, deletion of the $\mathrm{C}$-terminal tail results in a sequential relax- 

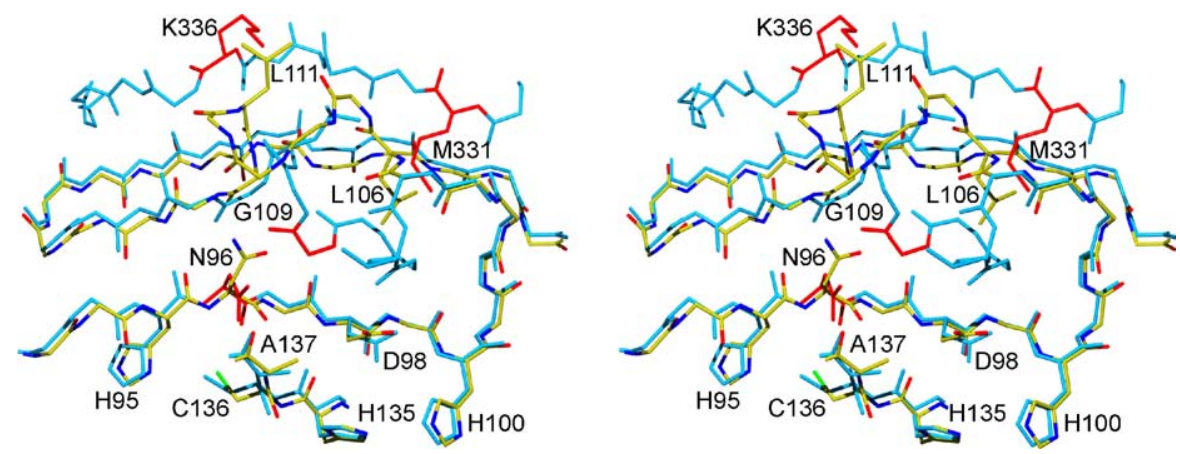

Fig. 3. Sequential steric constraining on active site geometry. Coordinates are taken from chain B of NiRc-11 (mixed color) and PDB entry 2 NRD of AcNiR (cyan). Superimposition is performed using Spdbviewer [29] based on the C $\alpha$ atoms of the whole trimer. Native enzyme residues involved in steric clash are highlighted in red. For clarity, side chains of unlabeled residues are omitted from the figure. (For interpretation of the references to color in this figure legend, the reader is referred to the web version of this paper.)

ation along the constraining chain, and finally leads to the distortion of the active site structure and the loss of the enzyme activity. The functional state of AcNiR is trimer. This study shows that the trimer formation not only completes the coordination of $\mathrm{T} 2 \mathrm{Cu}$ at the monomer interface, but also enables the final step modulation of the $\mathrm{T} 1 \mathrm{Cu}$ site geometry via sequential steric constraining. The AcNiR trimer structure provides an example of how the protein frameworks are designed for accommodating the copper ions and controlling their coordination.

\section{Hydrogen bond-fastened bulky residue motif in CuNiRs}

The discussions above clearly disclose the role of the Cterminal tail in modulating the geometries at the active site of AcNiR. Furthermore, the structural element responsible for such conformational modulation has been mapped to sequence segment 330-335 at the C-terminal. Superimposi- tion analysis reveals that Met331 plays a key role in constraining the irregular coil 105-112 with its bulky side chain (Fig. 3). It is shown in Fig. 4 that the C-terminal tail of $\mathrm{AcNiR}$ is anchored by forming an anti-parallel $\beta$-sheet with sequence segment $117-125$ in a different monomer. Altogether eleven hydrogen bonds are formed through the C-terminal tail, and five of them are retained in NiRc-5 [17], being the minimal hydrogen bonding motif responsible for the tight association. It is evident that there exists a hydrogen bond-fastened bulky residue motif in AcNiR, where the bulky residue Met331 is tightly fixed by at least five hydrogen bonds and protrudes its side chain against the irregular coil 105-112, providing the first layer of constraints on the active site structure.

Previous studies showed that the $\mathrm{CuNiR}$ family contains rather diverse members. According to the differences in spectroscopic property, CuNiRs have been classified into green copper, blue copper and other types [2]. Based on

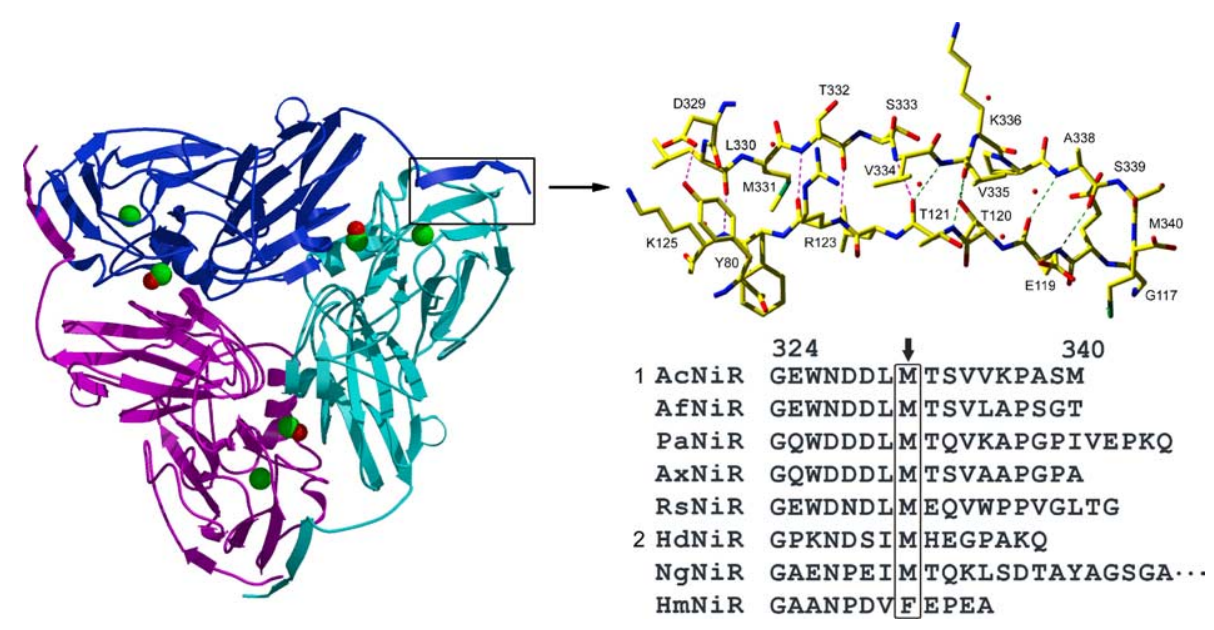

Fig. 4. Left: native AcNiR trimer (based on PDB entry 2NRD). One of the C-terminal tails is highlighted in rectangular box. Copper atoms and T2Cu ligating water are shown as green ball and small red ball, respectively. Right: hydrogen bond-fastened bulky residue motif among CuNiRs. Red dashes represent the minimal hydrogen bonding motif retained in NiRc-5. Bulky residue at position 331 is conserved among CuNiRs. Five class I and three class II members in the CuNiR family are shown here as an example. AcNiR, CuNiR from A. cycloclastes; AfNiR, CuNiR from A. faecalis; PaNiR, CuNiR from Pseudomonas aureofaciens; AxNiR, CuNiR from A. xylosocidans; RsNiR, CuNiR from R. sphaeroides; HdNiR, CuNiR from H. denitrificans; $\mathrm{NgNiR}, \mathrm{CuNiR}$ from Neisseria gonorrhoeae; $\mathrm{HmNiR}, \mathrm{CuNiR}$ from H. marismortui. (For interpretation of the references to color in this figure legend, the reader is referred to the web version of this paper.) 
the sequence homology, CuNiRs were divided into two classes recently [8]; a new class of CuNiRs (class II) characterized by the loss of two variable loops from the protein surface was identified in addition to the traditional class I members found mainly in the soil bacteria. Another interesting case is the CuNiR from Hyphomicrobium denitrificans ( $\mathrm{HdNiR})$, in which the common $\mathrm{CuNiR}$ becomes a major domain of the enzyme [26]. However, despite these diversities, sequence alignment shows that the bulky residue is well conserved at position 331 in CuNiRs (Fig. 4). In most species, the residue methionine is selected as the bulky residue; one exception is in the $\mathrm{HmNiR}$ from the archaebacterium Haloarcula marismortui, where the bulky residue phenylalanine is substituted for methionine. At the same time, certain length of the $\mathrm{C}$-terminal tail in $\mathrm{CuNiRs}$ is retained as well, which should correspond to the hydrogen bonds flanking the bulky residue. Interestingly, although being the shortest one in length, the C-terminal segment of $\mathrm{HmNiR}$ meets exactly the minimal hydrogen bonding motif mentioned above. Therefore, on the basis of homology analysis, it is strongly suggested that the hydrogen bond-fastened bulky residue motif is a common feature among the CuNiRs. Investigation of the solved $\mathrm{CuNiR}$ structures from other sources, including that of the class II NgNiR [8], reveals a similar configuration of Met331 and the crouched irregular coil in the protein, thus further confirming the essential role of the $\mathrm{C}$-terminal tail among the CuNiRs in maintaining an optimal active site geometry by the sequential steric constraining.

\section{Acknowledgments}

The project was supported by the National Key Research Development of China (Project No. G19990756 01), National Key Special Research Program (2002BA7 11A12), and Knowledge Innovation Program of Chinese Academy of Sciences (KJCS2-SW-N06). This work is dedicated to Prof. Jean Le Gall (1932-2003).

\section{References}

[1] W.G. Zumft, Cell biology and molecular basis of denitrification, Microbiol. Mol. Biol. Rev. 61 (1997) 533-616.

[2] S. Suzuki, K. Kataoka, K. Yamaguchi, T. Inoue, Y. Kai, Structurefunction relationships of copper-containing nitrite reductases, Coord Chem. Rev. 190-192 (1999) 245-265.

[3] J.W. Godden, S. Turley, D.C. Teller, E.T. Adman, M.Y. Liu, W.J. Payne, J. LeGall, The 2.3 angstrom X-ray structure of nitrite reductase from Achromobacter cycloclastes, Science 253 (1991) 438442.

[4] E.T. Adman, J.W. Godden, S. Turley, The structure of copper-nitrite reductase from Achromobacter cycloclastes at five $\mathrm{pH}$ values, with $\mathrm{NO}^{2-}$ bound and with type II copper depleted, J. Biol. Chem. 270 (1995) 27458-27474.

[5] M. Kukimoto, M. Nishiyama, M.E. Murphy, S. Turley, E.T. Adman, S. Horinouchi, T. Beppu, X-ray structure and site-directed mutagenesis of a nitrite reductase from Alcaligenes faecalis S-6: roles of two copper atoms in nitrite reduction, Biochemistry 17 (1994) 5246-5252.

[6] F.E. Dodd, J. Van Beeumen, R.R. Eady, S.S. Hasnain, X-ray structure of a blue-copper nitrite reductase in two crystal forms. The nature of the copper sites, mode of substrate binding and recognition by redox partner, J. Mol. Biol. 282 (1998) 369-382.

[7] T. Inoue, M. Gotowda, Deligeer, K. Kataoka, K. Yamaguchi, S. Suzuki, H. Watanabe, M. Gohow, Y. Kai, Type $1 \mathrm{Cu}$ structure of blue nitrite reductase from Alcaligenes xylosoxidans GIFU 1051 at $2.05 \AA$ resolution: comparison of blue and green nitrite reductases, J. Biochem. (Tokyo) 124 (1998) 876-879.

[8] M.J. Boulanger, M.E. Murphy, Crystal structure of the soluble domain of the major anaerobically induced outer membrane protein (AniA) from pathogenic Neisseria: a new class of copper-containing nitrite reductases, J. Mol. Biol. 315 (2002) 1111-1127.

[9] F. Jacobson, H. Guo, K. Olesen, M. Ökvist, R. Neutze, L. Sjölin, Structures of the oxidized and reduced forms of nitrite reductase from Rhodobacter sphaeroides 2.4 .3 at high $\mathrm{pH}$ : changes in the interactions of the type 2 copper, Acta Crystallogr. D 61 (2005) 1190-1198.

[10] R.W. Strange, L.M. Murphy, F.E. Dodd, Z.H. Abraham, R.R. Eady, B.E. Smith, S.S. Hasnain, Structural and kinetic evidence for an ordered mechanism of copper nitrite reductase, J. Mol. Biol. 287 (1999) 1001-1009.

[11] K. Kataoka, H. Furusawa, K. Takagi, K. Yamaguchi, S. Suzuki, Functional analysis of conserved aspartate and histidine residues located around the type 2 copper site of copper-containing nitrite reductase, J. Biochem. (Tokyo) 127 (2000) 345-350.

[12] M.J. Boulanger, M. Kukimoto, M. Nishiyama, S. Horinouchi, M.E. Murphy, Catalytic roles for two water bridged residues (Asp-98 and His-255) in the active site of copper-containing nitrite reductase, J. Biol. Chem. 275 (2000) 23957-23964.

[13] M.J. Boulanger, M.E. Murphy, Alternate substrate binding modes to two mutant (D98N and $\mathrm{H} 255 \mathrm{~N}$ ) forms of nitrite reductase from Alcaligenes faecalis S-6: structural model of a transient catalytic intermediate, Biochemistry 40 (2001) 9132-9141.

[14] J.G. Grossmann, Z.H. Abraham, E.T. Adman, M. Neu, R.R. Eady, B.E. Smith, S.S. Hasnain, X-ray scattering using synchrotron radiation shows nitrite reductase from Achromobacter xylosoxidans to be a trimer in solution, Biochemistry 32 (1993) 7360-7366.

[15] W.C. Chang, J.Y. Chen, T. Chang, M.Y. Liu, W.J. Payne, J. LeGall, W.C. Chang, The C-terminal segment is essential for maintaining the quaternary structure and enzyme activity of the nitric oxide forming nitrite reductase from Achromobacter cycloclastes, Biochem. Biophys. Res. Commun. 250 (1998) 782-785.

[16] T. Kakutani, H. Watanabe, K. Arima, T. Beppu, Purification and properties of a copper-containing nitrite reductase from a denitrifying bacterium, Alcaligenes faecalis strain S-6, J. Biochem. 89 (1981) 453461.

[17] H.T. Li, C. Wang, T. Chang, W.C. Chang, M.Y. Liu, J. LeGall, L.L. Gui, J.P. Zhang, X.M. An, W.R. Chang, pH-profile crystal structure studies of C-terminal despentapeptide nitrite reductase from Achromobacter cycloclastes, Biochem. Biophys. Res. Commun. 316 (2004) $107-113$.

[18] H.T. Li, T. Chang, M.Y. Liu, J. LeGall, X.M. An, L.L. Gui, J.P. Zhang, D.C. Liang, W.R. Chang, Preliminary crystallographic studies of two C-terminally truncated copper-containing nitrite reductases from Achromobacter cycloclastes: changed crystallizing behaviors caused by residue deletion, Biochem. Biophys. Res. Commun. 299 (2002) 173-176.

[19] Z. Otwinowski, W. Minor, Processing of X-ray diffraction data collected in oscillation mode, Methods Enzymol. 276 (1996) 307-326.

[20] J. Navaza, Amore: an automated package for molecular replacement, Acta Crystallogr. A 50 (1994) 157-163.

[21] A.T. Brunger, P.D. Adams, G.M. Clore, W.L. DeLano, P. Gros, R.W. Grosse-Kunstleve, J.S. Jiang, J. Kuszewski, M. Nilges, N.S. Pannu, R.J. Read, L.M. Rice, T. Simonson, G.L. Warren, Crystallography \& NMR system: a new software suite for macromolecular structure determination, Acta Crystallogr. D Biol. Crystallogr. 54 (1998) 905-921.

[22] G.J. Kleywegt, T.A. Jones, Where freedom is given, liberties are taken, Structure 3 (1995) 535-540. 
[23] T.A. Jones, J.Y. Zou, S.W. Cowan, Kjeldgaard, Improved methods for building protein models in electron density maps and the location of errors in these models, Acta Crystallogr. A 47 (1991) 110-119.

[24] O.H. Lowry, N.J. Rosebrough, A.L. Farr, R.J. Randall, Protein measurement with the folin phenol reagent, J. Biol. Chem. 193 (1951) 265-275.

[25] E. Libby, B.A. Averill, Evidence that the type 2 copper centers are the site of nitrite reduction by Achromobacter cycloclastes nitrite reductase, Biochem. Biophys. Res. Commun. 187 (1992) 1529-1535.

[26] K. Yamaguchi, K. Kataoka, M. Kobayashi, K. Itoh, A. Fukui, S. Suzuki, Characterization of two type $1 \mathrm{Cu}$ sites of Hypho- microbium denitrificans nitrite reductase: a new class of coppercontaining nitrite reductases, Biochemistry 43 (2004) 1418014188.

[27] P.J. Kraulis, MOLSCRIPT: a program to produce both detailed and schematic plots of protein structures, J. Appl. Crystallogr. 24 (1991) 946-950.

[28] E.A Merritt, D.J. Bacon, Raster3D: photorealistic molecular graphics, Methods Enzymol. 277 (1997) 505-524.

[29] N. Guex, M.C. Peitsch, SWISS-MODEL and the Swiss-PdbViewer: an environment for comparative protein modeling, Electrophoresis 18 (1997) 2714-2723. 